\title{
Detection of rice seed vigor by low-field nuclear magnetic resonance
}

\author{
Ping Song ${ }^{1,2,3}$, Peng Song ${ }^{2,3}$, Hongwei Yang ${ }^{1}$, Tao Yang ${ }^{1 *}$, Jing $\mathrm{Xu}^{1}$, Kaitian Wang ${ }^{1}$ \\ (1.College of Information and Electrical Engineering, Shenyang Agricultural University, Shenyang 110866, China; \\ 2. Beijing Research Center for Information Technology in Agriculture, Beijing 10097, China; \\ 3. National Research Center of Intelligent Equipment for Agriculture, Beijing 10097, China)
}

\begin{abstract}
A new method to predict the seed vigor of rice was developed to control adulteration during the seed trading process and to address the deficiencies of traditional manual detection methods. Low-field nuclear magnetic resonance (LF-NMR) technique was used to detect the vigor of rice seeds. Four varieties (Beijing-1, Qianchonglang-2, Yanfeng-47 and Shennong-265) of rice seeds from the Rice Research Institute of Shenyang Agricultural University were chosen for the experiment. The transverse relaxation time $T_{2}, T_{21}$ and $T_{22}$ were observed in the experiment. The peak start time of free water (transverse relaxation time $T_{22}$ ), signal amplitude of bound water (transverse relaxation time $T_{21}$ ), and moisture content decreased with the decrease in the vigor of the seeds. There were no obvious trends observed for the top of the peak and the end point of the transverse relaxation time $T_{22}$. In addition, the start, top, and end time of the peak (transverse relaxation time $T_{21}$ ), and the signal amplitude of bound water showed no consistent changes. The results indicated that LF-NMR could be used as a method to distinguish the vigor of rice seeds rapidly. This study provided theoretical basis and technical support for the rapid detection of rice seed vigor.
\end{abstract}

Keywords: nondestructive detection, nuclear magnetic resonance, transverse relaxation time, signal amplitude, rice, seeds vigor DOI: $10.25165 /$ j.ijabe. 20181106.4323

Citation: Song P, Song P, Yang H W, Yang T, Xu J, Wang K T. Detection of rice seed vigor by low-field nuclear magnetic resonance. Int J Agric \& Biol Eng, 2018; 11(6): 195-200.

\section{Introduction}

Seed vigor is an indicator of the integrative performance of the seed during the period of germination and emergence of the seedling. It is the potential ability of the seeds to grow quickly and develop into seedlings under the sowing conditions ${ }^{[1-3]}$. Rice is one of the main food crops, and the vigor level of the rice seed directly affects the seedling, which has an important influence on the yield of grain crops ${ }^{[4,5]}$. Adulteration was hard to detect and the fake seeds has the lower vigor, adulteration will result in heavy losses for famers. The main methods used for the detection of seed vigor are the direct method and the indirect method. The former is observed by simulating adverse conditions in the field to influence the speed of seedling growth, and the latter involves measuring some factors that are associated with seed vigor, such as physiological or biochemical indexes of the physical properties that indirectly determinate the seed vigor ${ }^{[6]}$. The ISTA handbook (ISTA procedure, third edition, 1995) for the determination of seed vigor recommends seven kinds of seed vigor testing methods, which use conductivity and accelerated aging as the measurement

\section{Receive date: 2018-04-12 Accepted date: 2018-09-17}

Biographies: Ping Song, $\mathrm{PhD}$, Associate Professor, research interest: precision agriculture, Email: songping_1010@163.com; Peng Song, Senior Engineer, research interest: plant information nondestructive acquisition technology and equipment, Email:26405974@qq.com; Hongwei Yang, Lecturer, research interest: precision agriculture, Email: 963344211@qq.com; Jing Xu, Lecturer, research interest: computer application, Email: 15101162@qq.com; Kaitian Wang, Senior Engineer, research interest: computer application, Email: 284788360@qq.com.

*Corresponding author: Tao Yang, PhD, Professor, research interest: precision agriculture. College of Information and Electrical Engineering, Shenyang Agricultural University, Shenyang 110866, China. Tel: +86-13840296051, Email: 328748306@qq.com. techniques. These detection methods have the advantage of being intuitive, but they have the disadvantages of manual operation, long operation time, subjective factors, extensive workload, and low efficiency $^{[7]}$. With the development of nondestructive testing technology, agricultural experts have increasingly performed various researches and achieved some remarkable results for seed vigor detection ${ }^{[8-14]}$. Kandpal et al. ${ }^{[15]}$ used Near-infrared hyperspectral imaging system predict viability and vigor of muskmelon seeds. Marchi et al. ${ }^{[16]}$ used software of seed vigor imaging system assess vigor of carrot seeds. Cheng et al. ${ }^{[17]}$ used the electronic nose to detect the germination rate of adulterated tomato seeds. The sampling results were classified and analyzed by back-propagation neural network. Zhu et al. ${ }^{[18]}$ analyzed the hardness characteristics of three varieties of soybean and cassia seeds by using near-infrared spectroscopy and established a qualitative analysis model. Wen et al. ${ }^{[19]}$ used electrospray extraction and ionization mass spectrometry to realize the determination of the seed vigor of soybean from different water extracts of soybean. Deng et al. ${ }^{[20]}$ used computer imaging technology to determine the length and growth rate of the seedlings of Chinese fir and Masson' pine and evaluate the seed vigor. However, the study of rice seed vigor using low-field nuclear magnetic resonance (LF-NMR) technique has not yet been reported This research uses LF-NMR technology to overcome the disadvantages of the above studies, which are low efficiency and dependence on artificial detection techniques such as electrical conductivity measurement and accelerated aging test. Rice seeds were used as the research object, and LF-NMR technology was used to detect the vigor of the rice seeds. The new method can quantitatively predict seed vigor and provides theoretical basis and technical support for the nondestructive testing of seed vigor. This research has high practicability and broad application prospect. 


\section{Materials and methods}

\subsection{Nuclear magnetic resonance imaging}

NMR is a nondestructive testing technology, which can be used to study the changes in the moisture distribution in the sample. NMR is based on the principle that the self-spin of the nucleus in the magnetic field follows the law $\omega_{0}=\gamma B_{0}\left(B_{0}\right.$ is the magnetic field intensity expressed in Tesla, $\gamma$ is the geomagnetic ratio, $\omega_{0}$ is the Larmor frequency expressed in megahertz $)^{[21,22]}$. The nucleus will undergo resonance when the radio frequency field is equal to the Larmor frequency. After the cessation of the RF pulse, the progression of the intensified self-spin nucleus from the state of activation to the state of equilibrium is called the relaxation process, and the time required for it is the relaxation time.

Different substances and different components of the same substance have different relaxation time in NMR testing, which can be utilized to distinguish between them ${ }^{[23]}$. In NMR spectroscopy, all hydrogen atoms in the sample will be excited by the hard radiofrequency pulse, thus generating a superposition relaxation signal that contains all the hydrogen protons. After obtaining the superimposed signal, the inverse operation of the NMR signal amplitude and the relaxation time are needed to distinguish between different components in the sample. The spectra obtained by the inverse operation are called the NMR inversion spectra.

\subsection{Materials}

In this study, MiniMR-60 NMR (magnet type: permanent magnets, magnetic field intensity: $(0.50 \pm 0.05) \mathrm{T}$, radio pulse frequency: $12.2 \mathrm{MHz}$, magnet temperature: $32^{\circ} \mathrm{C}$, probe coil diameter: $15 \mathrm{~mm}$; Shanghai new McLaren electronic technology Co., Ltd.) was used to measure the relaxation time $T_{2}$ and the signal amplitude of the sample. The tested rice varieties were Beijing-1, Qianchonglang-2, Yanfeng-47 and Shennong-265 provided by the Rice Research Institute of Shenyang Agricultural University in 2017.

\subsection{Methods}

\subsubsection{Sample preparation}

The rice seed varieties of Beijing-1, Qianchonglang-2, Yanfeng-47, and Shennong-265 were chosen for the experiment. The test sample comprised 3200 randomly selected mature seeds of each variety. The samples for each variety were divided into three groups of 200 grains, 1140 grains, and 1860 grains. The samples in the first group were used for the germination test to obtain the germination rate of normal-vigor seed according to Chinese National standard GB/T 3543.4-1995 $5^{[7]}$. The results of the germination test showed that the germination rate was nearly $100 \%$. The samples in the second group were placed in an electric blower drying box and continuously dried for $6 \mathrm{~h}$ at $80^{\circ} \mathrm{C}$ to obtain seeds without vigor. The seeds were stored under room temperature and humidity for seven days. The samples in the second group were added to samples of the third group, and five gradient aging groups with seed germination rates of $100 \%, 90 \%, 70 \%, 50 \%$ and $0 \%$ were obtained. Each gradient aging group contained 50 grains of each rice variety. The number of non-viable seeds in the five groups was $0,5,15,25$ and 50 . We could conclude that the corresponding germination rate was $100 \%, 90 \%, 70 \%, 50 \%$ and $0 \%$. Twelve parallel experiments were conducted for each group. The details of each group are shown in Table 1. The 240 samples $(4$ seed varieties $\times 5$ gradient aging groups $\times 12$ parallel experiments) were placed in a self-sealing bag.
Table 1 Grouping information of ageing rice seeds

\begin{tabular}{cccc}
\hline Variety & Classes & Grouping & $n$ /group \\
\hline Beijing-1 & non-glutinous rice & ABCDE & 50 \\
Qianchonglang-2 & non-glutinous rice & ABCDE & 50 \\
Yanfeng-47 & non-glutinous rice & ABCDE & 50 \\
Shennong-265 & non-glutinous rice & ABCDE & 50 \\
\hline
\end{tabular}

Note: In the grouping of the ageing seeds, A contains 50 vigorous seeds with $100 \%$ seed germination rate; B contains 45 vigorous seed and 5 non-viable seeds, and the seed germination rate is $90 \%$; C contains 35 vigorous seeds and 15 non-viable seeds, and the seed germination rate is $70 \%$; D contains 25 vigorous seeds and 25 non-viable seeds, and the seed germination rate is $50 \%$; E contains 50 non-viable seeds, and the seed germination rate is $0 \%$.

\subsubsection{NMR spectroscopy}

To balance the volatile matter at the top, all the samples were placed in a confined space for $1 \mathrm{~h}^{[24]}$. Firstly, the samples were placed in a centrifuge tube of 2-mL capacity. Secondly, the centrifuge tube was placed at the bottom of glass tube of $12-\mathrm{mm}$ diameter. Finally, the glass tube was placed at the NMR center to acquire the NMR spectrum signal. The sample attenuation curve was obtained, and then the transverse relaxation time and NMR signal amplitude of the samples were determined. The test was repeated four times.

The CPMG pulse sequence parameters are as follows. Radio frequency signal principal value: $S F 1=12 \mathrm{MHz}$ (consistent with the magnet principal value); offset of radio frequency signal: $O 1=$ $212605.51 \mathrm{kHz}$ (very slight changes in each test); $90^{\circ}$ radiofrequency pulse width: $P 1=7.5 \mu \mathrm{s}$; number of sampling points $T D=163840 ;$ repeated sampling interval time: $T W=1000 \mathrm{~ms}$; pre-amplifier gain: $P R G=2 ; 180^{\circ}$ radiofrequency pulse width: $P 2=11.52 \mu \mathrm{s}$; echo number: $\mathrm{NECH}=2000$; wait time of repeated sampling $T E=0.41 \mathrm{~ms}$; receiver receives the signal frequency: $S W=200 \mathrm{kHz}$; starting point for the sampling: $R F D=0.02 \mathrm{~ms}$; analog gain: $R G 1=20 \mathrm{~dB}$; digital gain: $D R G 1=3$; data radius: $D R=1$; accumulative sampling number: $N S=32$.

\subsubsection{NMR spectrum inversion}

The '.pea' file collected in the NMR spectrum analysis software was accessed using NMR inversion software. The sampling data of the peak number, peak starting time, peak point time, peak end time, peak area, peak ratio, and total area of the samples were obtained through an inversion operation.

\subsection{Data processing}

This research uses the SPSS 20.0 software for processing the data from the NMR inversion software. The average and standard deviation of the transverse relaxation time $T_{2}$ of each peak starting time, peak point time, peak end time, and peak area were calculated. Finally, the post-processing data, which regard as the value of the sample NMR signal, were analyzed and processed.

\section{Results}

\subsection{Relationship between transverse relaxation time and vigor of rice seeds}

The multi-component characteristics of the NMR spectra relaxation time $T_{2}$ of four rice seed varieties of different vigor gradients are presented. There were two peaks in each $T_{2}$ inversion spectra. Based on the NMR principle, the relaxation time $T_{2}$ of protons in different environments is different. The shorter the relaxation time $T_{2}$, the higher the level of combination of water and matter, and the lower the degree of freedom of the protons. The longer the relaxation time $T_{2}$, the lower the combination of water and matter, and the higher the degree of freedom of the protons. Therefore, the relaxation time $T_{2}$ can 
indirectly indicate the free or bound state characteristics of water $^{[25-27]}$. The NMR $T_{2}$ relaxation spectrum for the Beijing-1 sample was obtained by randomly selecting one test sample for each vigor gradient, as shown in Figure 1. In Figure 1, each peak end time, which is $T_{2 i}$, indicates the transverse relaxation time of the $i$-th component. The internal moisture content of the rice seed can be divided into bound water and free water based on the differences in the transverse relaxation time of the NMR inversion spectrum.

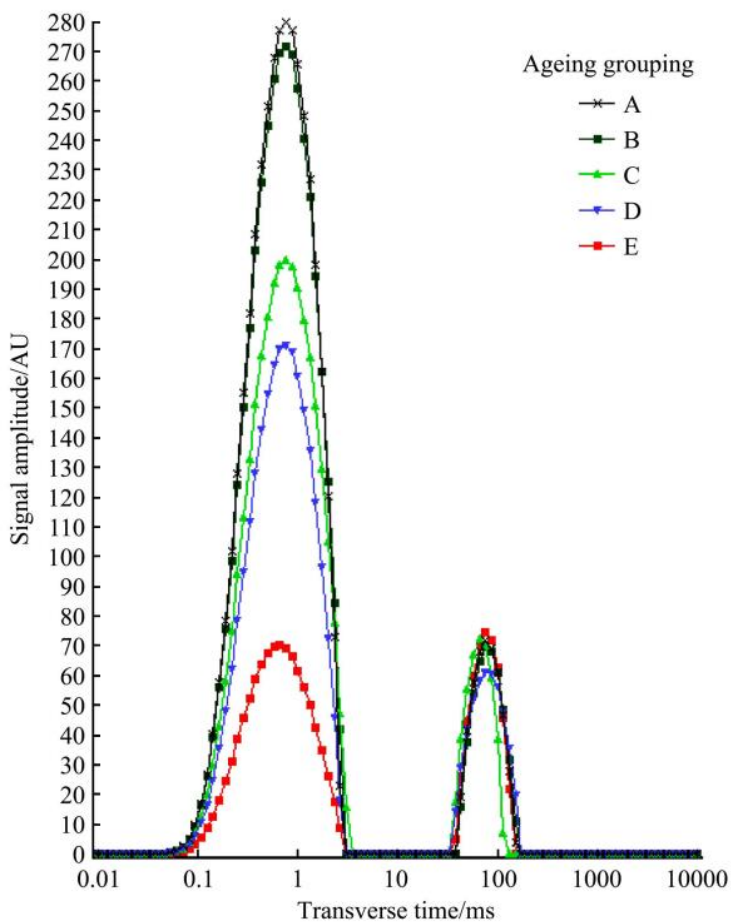

Note: Sample types: Beijing-1. In the grouping of the ageing seeds, A contains 50 vigorous seeds with $100 \%$ seed germination rate; B contains 45 vigorous seed and 5 non-viable seeds, and the seed germination rate is $90 \%$; C contains 35 vigorous seeds and 15 non-viable seeds, and the seed germination rate is $70 \%$; D contains 25 vigorous seeds and 25 non-viable seeds, and the seed germination rate is $50 \%$; E contains 50 non-viable seeds, and the seed germination rate is $0 \%$.

Figure 1 Spectrum inversion of transverse time $T_{2}$ of rice seeds

The short relaxation time $T_{21}(0.1-5 \mathrm{~ms})$ was an influencing factor for the bound water; the long relaxation time $T_{22}(20-200 \mathrm{~ms})$ was an influencing factor for the free water. The relaxation time $T_{2}$ indicates the chemical environment of the internal hydrogen proton. The larger the binding force of the hydrogen protons, the smaller the degree of freedom, the shorter the relaxation time $T_{2}$, and the closer the peak position of the relaxation spectrum $T_{2}$ to the left end of the axis. In contrast, the smaller the binding force of the hydrogen proton, the greater the degree of freedom, the longer the relaxation time $T_{2}$, and the closer the peak position of the relaxation spectrum $T_{2}$ to the right end of the axis. The peak start time, peak point time, and peak end time of the bound water of different vigor gradients (transverse relaxation time is $T_{21}$ ) do not follow an obvious trend. However, as shown in Table 2, the poorer the vigor of the rice seeds, the lager is the dispersion of the sampling data of the peak end time. It is not recommended that the use of this small change be regarded as the basis for differentiating the vigor of seeds.

This research observed the changes in the transverse relaxation time $T_{22}$ of free water through data analysis. As shown in Table 3, it was found that the poorer the vigor of the sample, the smaller is the initial time of the peak. This implies that the poorer the vigor of the sample, the weaker is the freedom of moisture content in the sample. However, the vigor of the sample at the peak of the transverse relaxation time $T_{22}$ and the end point did not follow an obvious trend.

Bound water and free water is in the living organisms exist in different proportions but have a dynamic balance. The different water ratios responsible for the different properties of various organisms ${ }^{[28-30]}$. The difference between free water and bound water is based on the strength of attraction between substances. The vigor of rice seeds can be detected by the difference in the transverse relaxation time of the samples, which were collected by NMR. We can distinguish between normal rice seeds and adulterated rice seeds based on the peak time of the transverse relaxation time $T_{22}$. The lower the vigor of rice seeds, the weaker is the freedom of the moisture content and the smaller is the initial time of the peak in the sample. The sampling time of the NMR test was approximately $40 \mathrm{~s}$. It is concluded that the LF-NMR technology can be used to realize the rapid detection of seed vigor according to the starting time of the peak of the transverse relaxation time $T_{22}$.

Table 2 Peak of transverse relaxation time $T_{21}$

\begin{tabular}{|c|c|c|c|c|c|}
\hline Variety & Germination rate & $n$ & Onset time of peak $/ \mathrm{ms}$ & Peak time/ms & End time of peak $/ \mathrm{ms}$ \\
\hline \multirow{5}{*}{ Beijing-1 } & $100 \%$ & 48 & 0.01 & 0.7565 & $3.1539 \pm 0.1920$ \\
\hline & $90 \%$ & 48 & 0.01 & $0.7534 \pm 0.1748$ & $3.1111 \pm 0.1537$ \\
\hline & $70 \%$ & 48 & 0.01 & $0.7600 \pm 0.0200$ & $3.1825 \pm 0.2089$ \\
\hline & $50 \%$ & 48 & 0.01 & $0.7226 \pm 0.0475$ & $3.2249 \pm 0.2249$ \\
\hline & $0 \%$ & 48 & 0.01 & $0.6071 \pm 0.0428$ & $2.9194 \pm 0.5906$ \\
\hline \multirow{5}{*}{ Qianchonglang-2 } & $100 \%$ & 48 & 0.01 & $0.7041 \pm 0.0500$ & $3.0700 \pm 0.1354$ \\
\hline & $90 \%$ & 48 & 0.01 & $0.7010 \pm 0.0497$ & $3.0452 \pm 0.1695$ \\
\hline & $70 \%$ & 48 & 0.01 & $0.6671 \pm 0.0292$ & $3.0060 \pm 0.1729$ \\
\hline & $50 \%$ & 48 & 0.01 & $0.6733 \pm 0.0363$ & $3.0737 \pm 0.2047$ \\
\hline & $0 \%$ & 48 & 0.01 & $0.6532 \pm 0.0400$ & $3.2444 \pm 0.6193$ \\
\hline \multirow{4}{*}{ Yanfeng-47 } & $100 \%$ & 48 & 0.01 & $0.7349 \pm 0.0414$ & $3.0682 \pm 0.0808$ \\
\hline & $90 \%$ & 48 & 0.01 & $0.7455 \pm 0.0316$ & $3.0244 \pm 0.1062$ \\
\hline & $70 \%$ & 48 & 0.01 & $0.7041 \pm 0.0450$ & $3.0682 \pm 0.0808$ \\
\hline & $50 \%$ & 48 & 0.01 & $0.6949 \pm 0.0485$ & $3.1129 \pm 0.1877$ \\
\hline \multirow{5}{*}{ Shennong-265 } & $100 \%$ & 48 & 0.01 & 0.7565 & $2.8711 \pm 0.2010$ \\
\hline & $90 \%$ & 48 & 0.01 & $0.7425 \pm 0.0325$ & $3.1254 \pm 0.3214$ \\
\hline & $70 \%$ & 48 & 0.01 & $0.7214 \pm 0.0311$ & $3.0214 \pm 0.1457$ \\
\hline & $50 \%$ & 48 & 0.01 & $0.6952 \pm 0.0325$ & $3.0587 \pm 0.1569$ \\
\hline & $0 \%$ & 48 & 0.01 & $0.6138 \pm 0.0435$ & $2.8867 \pm 0.5727$ \\
\hline
\end{tabular}

Note: All statistical data are measurement data expressed as mean \pm standard deviation $(\bar{x} \pm s)$. 
Table 3 Peak of transverse relaxation time $T_{22}$

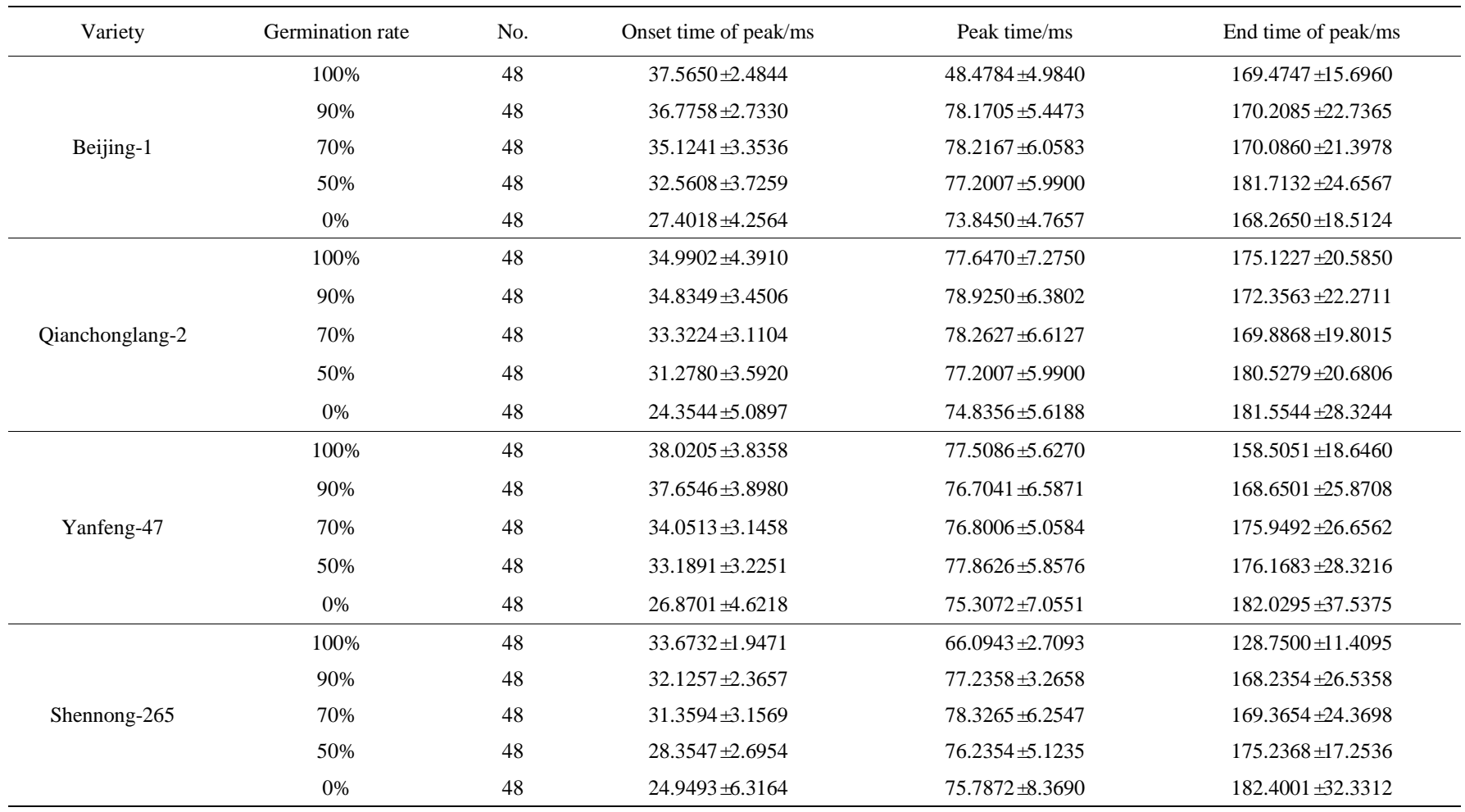

Note: All statistical data are measurement data expressed as mean \pm standard deviation $(\bar{x} \pm s)$

\subsection{Relationship between amplitude of NMR inversion spectrum and vigor of rice seeds}

The total amplitude of the $T_{2}$ relaxation spectrum from the NMR is $A$ ( $T_{2}$ relaxation spectrum corresponding to the totalpeak area of each peak). It is proportional to the number of hydrogen atoms in the sample. Therefore, the total $T_{2}$ relaxation spectrum amplitude ' $A$ ' reflects the overall moisture content in the samples. In this case, the signal amplitude of the transverse relaxation time $T_{21}$ is denoted by $A_{21}$, and the signal amplitude of the transverse relaxation time $T_{22}$ is denoted by $A_{22}$. $A$ represents the total moisture content of the rice seeds; then, $A=A_{21}+A_{22}$, where, $A_{21}$ indicates the bound water and $A_{22}$ indicates the free water content. 3.2.1 Effect of overall moisture content on seeds vigor.

Figure 2 shows the variation in the total water content of the rice seeds, which comprise four varieties of rice of different vigor gradients. The horizontal depicts axis the germination rate of the rice seeds of different vigor, and the vertical axis is the NMR signal amplitude corresponding to the relaxation time $T_{2}$. In Figure 2, the total moisture content of the four varieties of rice seeds decreases with the vigor of the seeds. This indicates that the samples with different germination rates $(100 \%, 90 \%, 70 \%, 50 \%$ and $0 \%$ ) can be distinguished by the variation in their total water content. In order to reduce the vigor of the seeds of rice and obtain the non-viable seeds, this experiment adopted the method of ultra-high temperature drying because the low moisture content can make the seeds lose their vigor. The higher the number of non-viable seeds, the lower is the amplitude of the MRI signal. This suggests that the NMR technology can be used as a new way to test the vigor of seeds.

Nevertheless, if we expose non-viable rice seeds to a damp environment, their inherent characteristic to absorb moisture from the surrounding environment increases the total moisture content of the seeds. This suggests that the change in the rice seed moisture content is not an accurate indicator of the overall vigor level.

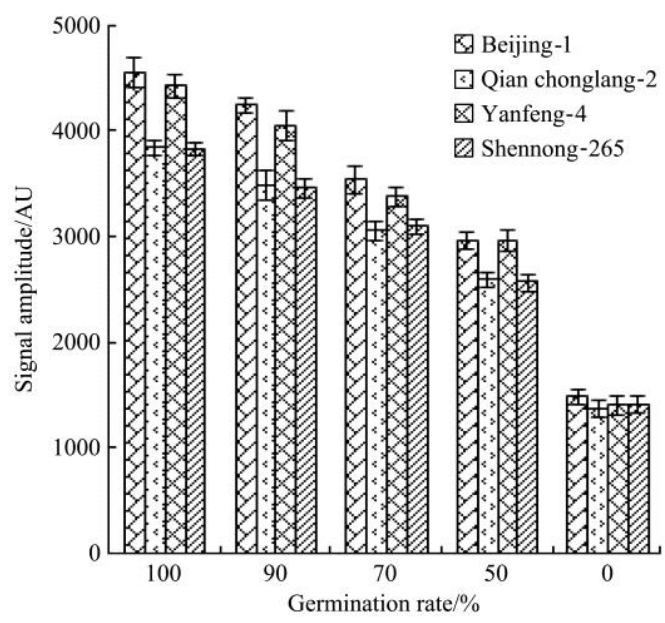

Figure 2 Changes in the signal amplitude of the overall moisture of rice seeds with diverse germination rates

3.2.2 Effect of free water content inside the seeds on seed vigor

Figure 3 shows the variation in the free water content for different varieties of rice seeds with varying vigor gradients. The horizontal axis shows the germination rates for the rice seeds of different vigor. The vertical axis is the signal amplitude of free water, which corresponds to the relaxation time $T_{22}$. There are no obvious rules for the variation in the freedom of internal water according to the decrease in the vigor of the seeds. It can be concluded that the signal amplitude of free water can not be used to differentiate the level of vigor of the seeds. When the seed is in a normal physical environment, moisture will be exchanged between the seed and the external environment. Therefore, the moisture content inside the seeds will change by the process of adsorption or evaporation. If the adsorption process prevails, the moisture in the seed will increase. If evaporation prevails, the moisture in the seed is reduced. If the seed is exposed to constant humidity and temperature, the seed water will stabilize after certain period of 
time and reach a state of equilibrium.

Free water is free to flow inside a living organism or cell, where it is useful as a solvent or as a means of transport. The rice seeds in this test were exposed to normal conditions for seven days after being dried at high temperature. Because of its characteristic of absorption moisture, the seeds absorb water from the environment, which then exists in form of free water inside the seeds. Therefore, in the experiment, we found that the content of free water in each group had little change. Even if the adulterated seeds are placed in a humid environment, the amount of water content in them can increase significantly but it can only be free water. Free water exists in the gap between the cell and tissue, and the binding force between the water and the seed colloid no longer exists. During the storage period, the increase in free water will not only promote seed deterioration but also decrease the vigor of the seeds.

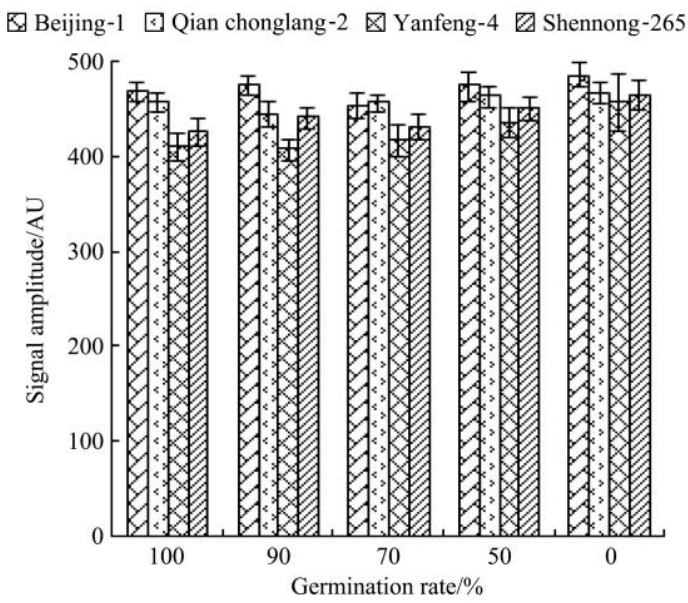

Figure 3 Variation in signal amplitude of water in rice seeds with diverse germination rates

\subsubsection{Effect of bound water inside the seeds on seed vigor}

Figure 4 shows the variation in the water content of the seeds with different vigor gradients for the four varieties of rice seeds. The horizontal axis shows the germination rates of rice seeds with different vigor gradients, and the vertical axis shows the signal amplitude of bound water corresponding to the NMR relaxation time is $T_{21}$. With the decrease in the vigor of the seeds, the bound water content of seeds shows a significant downward trend. By comparing Figures 2 and 4, it can be concluded that the main contributor to the change in the overall water content of the seeds is the bound water. This indicates that the vigor levels of rice seed samples can be distinguished by their bound water content (transverse relaxation time is $T_{21}$ ). The bound water is a combination of hydrogen bond and the polar base of protein, which adsorbs and binds to the organic solid material by forming hydrocolloids. Free water changes to bound water only when the metabolism of the seeds is active, which results in the increase in the bound water content; the internal bound water content will be lower for the rice seed with lesser vigor.

The moisture content in the seed consists of free water and the bound water, and the content of free water is greatly influenced by external environment factors. Therefore, the variation in the signal amplitude of the bound water is a better evaluation factor for analyzing the vigor of seeds than the change in the total water content. The state of the internal water can be measured from the NMR spectra, which can be used to identify the usefulness of the seeds according to the changes in the different states of water.

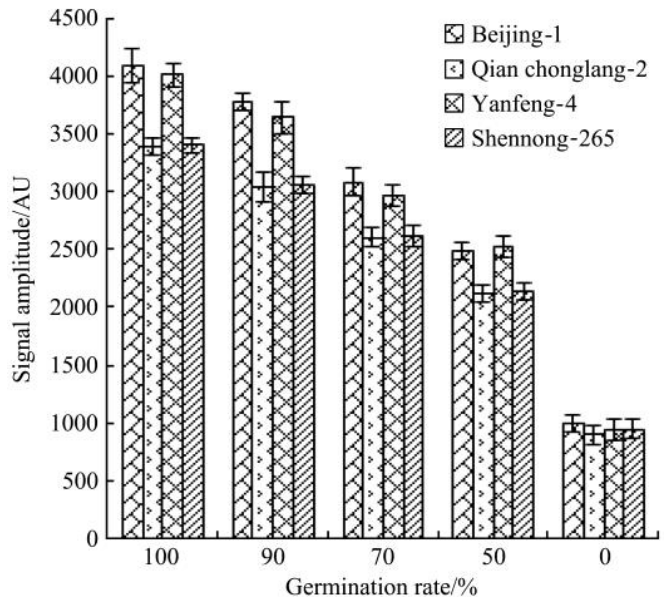

Figure 4 Variation in the signal amplitude of overall water content in rice seeds with diverse germination rates

\subsection{Relationship between moisture content ratio and vigor of} rice seeds

Figure 5 depicts the proportions of free water and bound water in the four varieties of rice seeds with different vigor gradient. It is observed that with the decrease in the level of vigor of the rice seeds, the proportion of bound water in the total water content shows a decreasing trend, whereas the proportion of free water in the total water content shows an upward trend.

Free water acts as a solvent and enhances the metabolic activity; moreover, the cell membranes have high water permeability because of which water has high movability and is easily lost from the rice seeds. The free water of seeds with low vigor can be supplemented with water from the external environment. However, water can not combine with the internal constituents of the rice seeds; the external water cannot be absorbed by the colloidal particles or large molecules in the plant cells, and can not participate in biochemical reactions. Therefore, the bound and free water ratio can also be used as the factor for determining the vigor level of rice seeds.
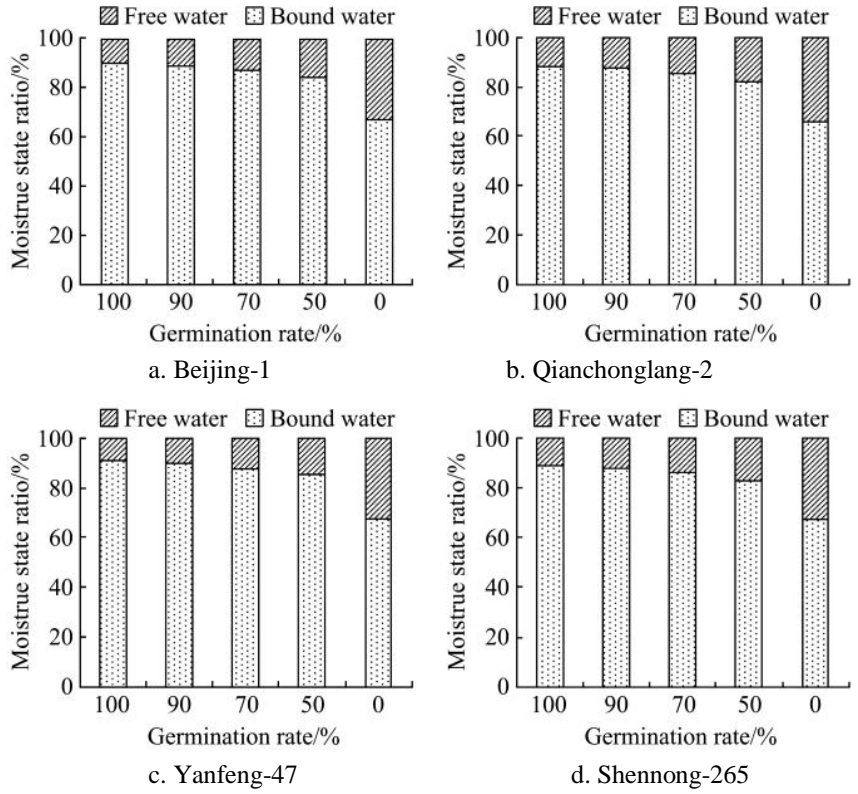

Figure 5 Proportions of bound water and free water in the seeds with diverse germination rates

\section{Discussion}

By using the NMR technology, the signal values of rice seeds 
with five gradient vigor groups which germination rate of $100 \%$, $90 \%, 70 \%, 50 \%$ and $0 \%$ were collected. Through data analysis, it was found that the transverse relaxation time of the NMR inversion spectrum and the signal amplitude were different. This shows that the LF-NMR technology can be used to quickly distinguish the adulteration of rice seeds.

By analyzing transverse relaxation time, it was found that the peak starting time, peak time, and peak end time (transverse relaxation time is $T_{21}$ ) of bound water, and the peak time and peak end time (transverse relaxation time is $T_{22}$ ) of free water have no obvious differences. The peak start time of free water decreases with the decrease in the vigor of the seeds, which indicates that the vigor level of seeds can be detected by the position of peak start time with transverse relaxation time $T_{22}$

The poorer the level of vigor, the lower is the moisture content in the rice seed. However, the reduction in the water content of the seeds is mostly through loss of bound water, and the free water content shows a small change. If the adulterated rice seeds are placed in a damp environment, only the free water content will increase whereas the bound water will not change; therefore, the NMR signal amplitude of bound water can used as a way to detect the rice seed vigor level, the test results of which are directly and clearly.

\section{Acknowledgement}

The project was supported by National Natural Science Foundation of China (Grant No.31701318 and 31601216), National Natural Science Foundation of China Projects of International Cooperation and Exchanges (Grant No. 31811540396), National Key Research and Development Program of China (Grant No.2017YFD0701205), and Doctoral Research Fund of Liaoning Province, China (Grant No.20170520202).

\section{[References]}

[1] Wiensczyk A, Swift K, Morneault A, Thiffault N, Szuba K, Wayne B. An overview of the efficacy of vegetation management alternatives for conifer regeneration in boreal forests. The Forestry Chronicle, 2011; 87(2): 175-200

[2] Oakley K, Geneve R, Siriwitayawan G, Kester S. Use of digital analysis of radicle extension of marigold seedlings as an early indicator of seed vigor. Hort Science A Publication of the American Society for Horticultural Science, 1998; 33(3): 467.

[3] Jilani G, Saxena R C, Khan A A. Ethylene production as an indicator of germination and vigor loss in stored rice seed infested by Rhyzopertha dominica, (F.) (Coleoptera: Bostrychidae). Journal of Stored Products Research, 1989; 25(3): 175-178.

[4] Jia J, Wang J H, Xie Z M, Yang L M, Sun B Q, Sun Q. Wheat seeds selection based on computer image recognition technique. Journal of China Agricultural University, 2014; 19(5): 180-186. (in Chinese)

[5] Wang J, Hu X W, He X Q, Wang Y R. The characteristics of germination and seedling emergence of several grasses seeds with different vigor. Pratacultural Science, 2011; 28(6): 998-1003.

[6] Han J, Mao P, Pu X, Li M. A study on the seed vigour of tall fescue. Acta Agrestia Sinica, 1995; 34(4): 269-275.

[7] Rules for agricultural seed testing - Germination test.GB/T 3543.4-1995.

[8] Soltani A, Lestander T, Tigabu M, Odén P. Prediction of viability of oriental beechnuts, Fagus orientalis, using near infrared spectroscopy and partial least squares regression. Journal of Near Infrared Spectroscopy, 2003; 11(5): 357-364.

[9] Tigabu M, Odén P C. Discrimination of viable and empty seeds of Pinus patula, Schiede \& Deppe with near-infrared spectroscopy. New Forests,
$2003 ; 25(3): 163-176$

[10] Wei L, Zhang X, Zhang B, Li F. Electronic nose technology and its application in the activity detection of wheat grain. Journal of Agricultural Mechanization Research, 2009; 6: 191-193. (in Chinese)

[11] Yin S X, Yang D F, Wang X Z, Gao S R, Jiang L. Application study of near infrared spectroscopy technology in maize seed vigor detection. Modern Agricultural Science \& Technology, 2015; 13: 20-23. (in Chinese)

[12] Chen R J, Zhao G W, Cao D D, Guan-Hai R, Chen H Y. The application of oxygen sensing technology in rapid vigor testing of treated conventional rice seeds. Seed, 2013; 32(5):8-16 (in Chinese)

[13] Xu J, Guo S, Jia G, Zhang N, Song Z, Xu G, et al. Effect of corona discharge field processing on vigor of three layers of paddy seeds. Transactions of the CSAE, 2015; 31(Z1): 307-314. (in Chinese)

[14] Wu W, Xia Y, Zhou D, Zhao X, Chen X. Relationship between biological superweak luminescence and viability of post-drying maize seeds. Transactions of the CSAE, 2002; 18(3): 8-10. (in Chinese)

[15] Kandpal L M, Lohumi S, Kim M S, Kang J S, Cho B K. Near-infrared hyperspectral imaging system coupled with multivariate methods to predict viability and vigor in muskmelon seeds. Sensors \& Actuators B Chemical, 2016; 229: 534-544.

[16] Marchi J L D, Cicero S M, Marchi J L D, Cicero S M. Use of the software seed vigor imaging system (SVIS®) for assessing vigor of carrot seeds. Sci. Agric, 2017; 74(6): 469-473.

[17] Cheng S, Wang J, Ma Y, Wang Y, Wei Z. Detection of germination rate of tomato seeds by electronic nose. Transactions of the CSAE, 2011; 27(12): 132-135. (in Chinese)

[18] Zhu L, Huang Y, Wang Q, Ma H, Sun B, Sun Q. Nondestructive identification of hard seeds of three legume plants using near infrared spectroscopy. Transactions of the CSAE, 2012; 28: 237-242. (in Chinese)

[19] Wen Z, Luo H, Guo X, Huang X, Hua C, Luo L. Rapid identification of seed vigor of soybean water extracts by electrospray ionization mass spectrometry. Transactions of the CSAE, 2016; 32(16): 273-278. (in Chinese)

[20] Deng F, Qi H, Zhao G. Seed vigor assessment for Cunninghamia lanceolata and Pinus massoniana using image processing. Transactions of the CSAE, 2012; 28: 274-279. (in Chinese)

[21] Choi S G, Kerr W L. ${ }^{1}$ H NMR studies of molecular mobility in wheat starch. Food Research International, 2003; 36(4): 341-348.

[22] Chen F L, Wei Y M, Zhang B. Characterization of water state and distribution in textured soybean protein using DSC and NMR. Journal of Food Engineering, 2010; 100(3): 522-526. (in Chinese)

[23] Gu Z, Liu W. The inversion of two-dimensional NMR map. Chinese Journal of Magnetic Resonance, 2007; 24(3): 311-319. (in Chinese)

[24] Pang L J, Wang J. Discrimination of different storage time of the wheat by electronic nose. Chinese Journal of Sensors \& Actuators, 2007; 20(8): 1717-1722. (in Chinese)

[25] Song P, Xu J, Ma H, Wang C, Yang T, Gao H. Moisture phase state and distribution characteristics of seed during rice seed soaking process by low field nuclear magnetic resonance. Transactions of the CSAE, 2016; 32(6) 204-210. (in Chinese)

[26] Song P, Yang T, Wang C, Song P, Pan D. Effects of rice seed soaking methods on moisture absorption capacity by low-field nuclear magnetic resonance. Transactions of the CSAE, 2016; 32(7): 237-243. (in Chinese)

[27] Song P, Yang T, Wang C, Pan D, Ren P. Analysis of moisture changes during rice seed soaking process using low-field NMR. Transactions of the CSAE, 2015; 31(15): 279-284. (in Chinese)

[28] Hinrichs R, Gotz J, Noll M, Wolfschoon A, Eibel H, Weisser H. Characterisation of the water-holding capacity of fresh cheese samples by means of low resolution nuclear magnetic resonance. Food Research International, 2004; 37(7): 667-676.

[29] Han M, Zhang Y, Ying F, Xu X, Zhou G. Effect of microbial transglutaminase on NMR relaxometry and microstructure of pork myofibrillar protein gel. European Food Research \& Technology, 2009; 228(4): 665-670.

[30] Yang W, Zhang W, Wang X, Xu D, Lou Q, Zhang J, et al. Effect of salt solution rinse on properties of hairtail surimi gel by low-field nuclear magnetic resonance. Transactions of the CSAE, 2016; 32(7): 263-269. (in Chinese) 